\title{
A COMPLETELY MONOTONIC FUNCTION INVOLVING THE DIVIDED DIFFERENCE OF THE PSI FUNCTION AND AN EQUIVALENT INEQUALITY INVOLVING SUMS
}

\author{
FENG QI ${ }^{12}$ \\ (Received 13 November, 2006)
}

\begin{abstract}
In this paper, a function involving the divided difference of the psi function is proved to be completely monotonic, a class of inequalities involving sums is found, and an equivalent relation between complete monotonicity and one of the class of inequalities is established.
\end{abstract}

2000 Mathematics subject classification: primary 33B15, 26D15; secondary 26A48, $26 \mathrm{~A} 51$.

Keywords and phrases: completely monotonic function, psi function, polygamma function, inequality, sum, equivalence.

\section{Introduction}

It is well known that the classical Euler's gamma function $\Gamma(x)$ is one of the most important special functions and has extensive applications in many fields of science, for example, in statistics, physics, engineering and the mathematical sciences. The logarithmic derivative of $\Gamma(x)$, denoted by $\psi(x)=\Gamma^{\prime}(x) / \Gamma(x)$, is called the psi or digamma function, and the derivatives $\psi^{(i)}(x)$ for $i \in \mathbb{N}$ of the psi function $\psi(x)$ are known as the polygamma or multigamma functions.

A function $f$ is said to be completely monotonic on an interval $l$ if $f$ has derivatives of all orders on $I$ and $0 \leq(-1)^{n} f^{(n)}(x)<\infty$ for $x \in I$ and $n \geq 0$ [11]. A positive function $f$ is called logarithmically completely monotonic on an interval $I$ if $f$ has derivatives of all orders on $I$ and its logarithm $\ln f$ satisfies $0 \leq(-1)^{k}[\ln f(x)]^{(k)}<\infty$ for all $k \in \mathbb{N}$ on $I$. The set of the completely monotonic functions on $I$ is denoted

\footnotetext{
${ }^{1}$ College of Mathematics and Information Science, Henan University, Kaifeng City, Henan Province, 475001, China.

${ }^{2}$ Research Institute of Mathematical Inequality Theory, Henan Polytechnic University, Jiaozuo City, Henan Province, 454010, China; e-mail: qifeng618@gmail.com, qifeng618@hotmail.com (C) Australian Mathematical Society 2007, Serial-fee code 1446-1811/07
} 
by $\mathscr{C}[I]$ and the set of the logarithmically completely monotonic functions on $I$ is denoted by $\mathscr{L}[I]$. It is proved in $[3,11,13,16]$ that $\mathscr{L}[I] \subset \mathscr{C}[I]$. The wellknown Bernstein's Theorem [17, page 161] states that $f \in \mathscr{C}[(0, \infty)]$ if and only if $f(x)=\int_{0}^{\infty} e^{-x s} d \mu(s)$, where $\mu$ is a nonnegative measure on $[0, \infty)$ such that the integral converges for all $x>0$. This expresses that $f \in \mathscr{C}[(0, \infty)]$ is a Laplace transform of the measure $\mu$. In $[3$, Theorem 1.1] and $[8,15]$ it is pointed out that the logarithmically completely monotonic functions on $(0, \infty)$ can be characterized as the infinitely divisible completely monotonic functions. In the recent past, numerous functions, which are defined in terms of the gamma, polygamma, and other special functions, have been proved to be (logarithmically) completely monotonic and this fact is used to derive many interesting new inequalities (see, for example, [1,3$6,8,11,14-16]$ and the references therein).

Kershaw's inequality [9] states that

$$
\left(x+\frac{s}{2}\right)^{1-s}<\frac{\Gamma(x+1)}{\Gamma(x+s)}<\left(x-\frac{1}{2}+\sqrt{s+\frac{1}{4}}\right)^{1-s}
$$

for $0<s<1$ and $x \geq 1$, which improved the corresponding result in [7]. In $[4,6,10,14]$, in order to obtain the best upper and lower bounds for the double inequality (1.1), the monotonicity and convexity properties of the function

$$
\left[\frac{\Gamma(x+t)}{\Gamma(x+s)}\right]^{1 /(t-s)}-x
$$

in $x \in(-\alpha, \infty)$ are verified, where $s$ and $t$ are nonnegative numbers and $\alpha=\min \{s, t\}$. In $[4,6,10,14]$, in order to prove the monotonicity and convexity of $(1.2)$, the function

$$
\delta_{s, t}(x)= \begin{cases}\frac{\psi(x+t)-\psi(x+s)}{t-s}-\frac{2 x+s+t+1}{2(x+s)(x+t)}, & s \neq t \\ \psi^{\prime}(x+s)-\frac{1}{x+s}-\frac{1}{2(x+s)^{2}}, & s=t\end{cases}
$$

in $x \in(-\alpha, \infty)$, involving the divided difference of the psi function, is derived. Recently, the positivity of the function $\delta_{0,0}(x)=\psi^{\prime}(x)-1 / x-1 / 2 x^{2}$ was proved in $[2,5,12]$ respectively.

The first aim of this paper is to prove the complete monotonicity property of the function $\delta_{s, t}(x)$.

THEOREM 1.1. Let $s$ and $t$ be nonnegative numbers and $\alpha=\min \{s, t\}$. Then the functions $\delta_{s, t}(x)$ for $|t-s|<1$ and $-\delta_{s, t}(x)$ for $|t-s|>1$ are completely monotonic in $x \in(-\alpha, \infty)$. 
The second aim of this paper is to found a class of inequalities involving sums.

THEOREM 1.2. Let $k$ be a nonnegative integer and $\theta>0$ a constant. If $a>0$ and $b>0$, then

$$
\sum_{i=0}^{k} \frac{1}{(a+\theta)^{i+1}(b+\theta)^{k-i+1}}+\sum_{i=0}^{k} \frac{1}{a^{i+1} b^{k-i+1}}>2 \sum_{i=0}^{k} \frac{1}{(a+\theta)^{i+1} b^{k-i+1}}
$$

holds for $b-a>-\theta$ and the inequality is reversed for $b-a<-\theta$.

If $a<-\theta$ and $b<-\theta$, then the inequalities

$$
\sum_{i=0}^{2 k} \frac{1}{(a+\theta)^{t+1}(b+\theta)^{2 k-t+1}}+\sum_{i=0}^{2 k} \frac{1}{a^{i+1} b^{2 k-i+1}}>2 \sum_{i=0}^{2 k} \frac{1}{(a+\theta)^{i+1} b^{2 k-i+1}}
$$

and

$$
\sum_{i=0}^{2 k+1} \frac{1}{(a+\theta)^{t+1}(b+\theta)^{2 k-t+2}}+\sum_{i=0}^{2 k+1} \frac{1}{a^{i+1} b^{2 k-i+2}}<2 \sum_{i=0}^{2 k+1} \frac{1}{(a+\theta)^{i+1} b^{2 k-i+2}}
$$

hold for $b-a>-\theta$ and the reverse holds for $b-a<-\theta$.

If $-\theta<a<0$ and $-\theta<b<0$, then inequality (1.4) holds and inequality (1.5) is valid for $a+b+\theta>0$ and is reversed for $a+b+\theta<0$.

If $a<-\theta$ and $b>0$, then inequality (1.4) holds and inequality (1.5) is valid for $a+b+\theta>0$ and is reversed for $a+b+\theta<0$.

If $a>0$ and $b<-\theta$, then inequality (1.4) is reversed and inequality (1.5) holds for $a+b+\theta<0$ and reverses for $a+b+\theta>0$.

If $b=a-\theta$, then inequalities (1.3), (1.4) and (1.5) become equalities.

REMARK 1. The shaded areas in the diagram below describe the domains on the plane $a O b$ on which Theorem 1.2 is valid.

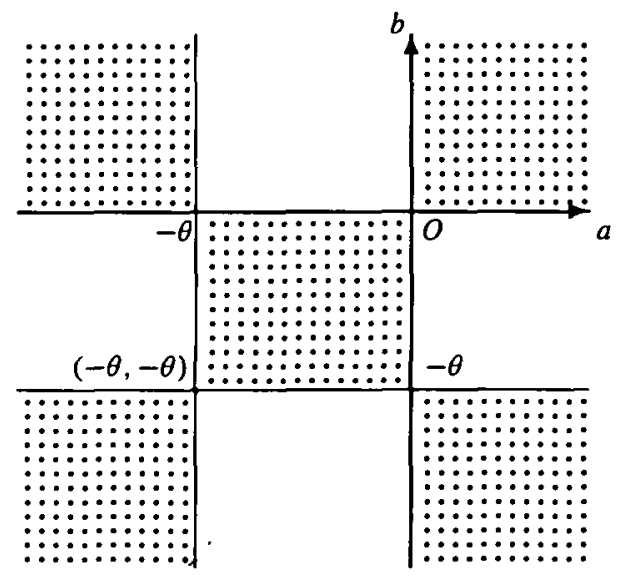


REMARK 2. Hinted at by Theorem 1.2, the following open problem is posed: Let $k$ be a nonnegative integer and $\theta$ a positive constant. Discuss the validity of the inequality

$$
\frac{(a+b)^{k}}{a^{k+1} b^{k+1}}+\frac{(a+b+2 \theta)^{k}}{(a+\theta)^{k+1}(b+\theta)^{k+1}}>\frac{2(a+b+\theta)^{k}}{(a+\theta)^{k+1} b^{k+1}}
$$

or its reverse for $a, b \notin\{0,-\theta\}$.

The final aim of this article is to establish an equivalent relationship between Theorem 1.1 and inequality (1.3) for positive numbers $a$ and $b$.

THEOREM 1.3. Inequality (1.3) for positive $a$ and $b$ is equivalent to Theorem 1.1.

\section{Proofs of theorems}

The following lemma is fundamental to this paper.

LEMMA 2.1. Let $f(x)$ be defined on an infinite interval I. If $\lim _{x \rightarrow \infty} f(x)=0$ and $f(x)-f(x+\varepsilon)>0$ for any given $\varepsilon>0$, then $f(x)>0$ on $I$.

ProOF. By induction, for any $x \in I$, we have

$$
f(x)>f(x+\varepsilon)>f(x+2 \varepsilon)>\cdots>f(x+k \varepsilon) \rightarrow 0
$$

as $k \rightarrow \infty$. The proof of Lemma 2.1 is complete.

PROOF OF THEOREM 1.1. (1) Case $\delta_{s, s}(x)$. We only need to prove that the function $\delta_{c, 0}(x)=\psi^{\prime}(x)-1 / x-1 / 2 x^{2}$ is completely monotonic on $(0, \infty)$.

Successive differentiation of the function $\delta_{0,0}(x)$ with respect to $x>0$ yields

$$
\delta_{0,0}^{(k)}(x)=\psi^{(k+1)}(x)+\frac{(-1)^{k+1} k !}{x^{k+1}}+\frac{(-1)^{k+1}(k+1) !}{2 x^{k+2}}
$$

for a nonnegative integer $k$.

It is well known that the polygamma functions $\psi^{(n)}(x)$ can be expressed as

$$
\begin{aligned}
\psi^{(n)}(x) & =(-1)^{n+1} \int_{0}^{\infty} \frac{t^{n}}{1-e^{-t}} e^{-x t} d t \quad \text { for } n \in \mathbb{N}, \quad \text { and } \\
\frac{1}{x^{r}} & =\frac{1}{\Gamma(r)} \int_{0}^{\infty} t^{r-1} e^{-x t} d t \quad \text { for } x>0 \text { and } r>0 .
\end{aligned}
$$

Applying formulae (2.2) and (2.3) in (2.1) yields

$$
(-1)^{k} \delta_{0,0}^{(k)}(x)=\int_{0}^{\infty}\left(\frac{t}{1-e^{-t}}-1-\frac{t}{2}\right) t^{k} e^{-x t} d t>0 .
$$


Thus the function $\delta_{0,0}(x)$ is completely monotonic in $(0, \infty)$.

(2) Case $\delta_{s, t}(x)$ for $s \neq t$. The function $\delta_{s, t}(x)$ can be rewritten as

$$
\delta_{s, t}(x)=\frac{1}{t-s} \int_{s}^{t} \psi^{\prime}(x+u) d u-\frac{1}{2}\left[\left(1-\frac{1}{t-s}\right) \frac{1}{x+t}+\left(1+\frac{1}{t-s}\right) \frac{1}{x+s}\right],
$$

so, for nonnegative integer $k$,

$$
\begin{aligned}
\delta_{s, t}^{(k)}(x)= & \frac{1}{t-s} \int_{s}^{t} \psi^{(k+1)}(x+u) d u \\
& -\frac{(-1)^{k} k !}{2}\left[\left(1-\frac{1}{t-s}\right) \frac{1}{(x+t)^{k+1}}+\left(1+\frac{1}{t-s}\right) \frac{1}{(x+s)^{k+1}}\right] .
\end{aligned}
$$

Since $\lim _{x \rightarrow \infty} \delta_{s, t}^{(k)}(x)=0$ from (2.2), by Lemma 2.1 , to show $(-1)^{k} \delta_{s, t}^{(k)}(x) \gtrless 0$, it is sufficient to verify $(-1)^{k}\left[\delta_{s, l}^{(k)}(x)-\delta_{s, t}^{(k)}(x+1)\right] \gtrless 0$.

Taking the logarithm of the difference equation $\Gamma(x+1)=x \Gamma(x)$ and consecutively differentiating yields

$$
\psi^{(i-1)}(x+1)=\psi^{(i-1)}(x)+\frac{(-1)^{t-1}(i-1) !}{x^{i}}
$$

for $i \in \mathbb{N}$ and $x>0$.

In the following, for convenience and simplicity, denote $p=x+s>0$ and $q=x+t>0$, when there is no confusion in the context.

Using formulae (2.3) and (2.4), we obtain

$$
\begin{aligned}
&(-1)^{k}\left[\delta_{s, t}^{(k)}(x)-\delta_{s, t}^{(k)}(x+1)\right] \\
&=\frac{(-1)^{k}}{t-s} \int_{s}^{t}\left[\psi^{(k+1)}(x+u)-\psi^{(k+1)}(x+u+1)\right] d u \\
& \quad-\frac{k !}{2}\left\{\left[1-\frac{1}{t-s}\right]\left[\frac{1}{q^{k+1}}-\frac{1}{(q+1)^{k+1}}\right]\right. \\
&\left.\quad+\left[1+\frac{1}{t-s}\right]\left[\frac{1}{p^{k+1}}-\frac{1}{(p+1)^{k+1}}\right]\right\} \\
&=\frac{(k+1) !}{t-s} \int_{s}^{t} \frac{1}{(x+u)^{k+2}} d u-\frac{k !}{2}\left\{\left[1-\frac{1}{t-s}\right]\left[\frac{1}{q^{k+1}}-\frac{1}{(q+1)^{k+1}}\right]\right. \\
&\left.+\left[1+\frac{1}{t-s}\right]\left[\frac{1}{p^{k+1}}-\frac{1}{(p+1)^{k+1}}\right]\right\} \\
&=\frac{k !}{t-s}\left[\frac{1}{p^{k+1}}-\frac{1}{q^{k+1}}\right]-\frac{k !}{2}\left\{\left[1-\frac{1}{t-s}\right]\left[\frac{1}{q^{k+1}}-\frac{1}{(q+1)^{k+1}}\right]\right. \\
&\left.+\left[1+\frac{1}{t-s}\right]\left[\frac{1}{p^{k+1}}-\frac{1}{(p+1)^{k+1}}\right]\right\}
\end{aligned}
$$




$$
\begin{aligned}
= & \frac{k !}{2}\left\{\left(1+\frac{1}{q-p}\right) \frac{1}{(p+1)^{k+1}}+\left(\frac{1}{q-p}-1\right) \frac{1}{p^{k+1}}\right. \\
& \left.+\left(1-\frac{1}{q-p}\right) \frac{1}{(q+1)^{k+1}}-\left(1+\frac{1}{q-p}\right) \frac{1}{q^{k+1}}\right\} \\
= & \frac{1}{2} \int_{0}^{\infty} z^{k}\left[\left(1+\frac{1}{q-p}\right) e^{-(p+1) z}+\left(\frac{1}{q-p}-1\right) e^{-p z}\right. \\
& \left.+\left(1-\frac{1}{q-p}\right) e^{-(q+1) z}-\left(1+\frac{1}{q-p}\right) e^{-q z}\right] d z \\
= & \frac{1}{2} \int_{0}^{\infty} z^{k+1}\left(e^{z}+1\right)\left[e^{-(p+1) z}+e^{-(q+1) z}\right] \\
& \times\left[\frac{1}{(q-p) z} \frac{e^{(q-p) z}-1}{e^{(q-p) z}+1}-\frac{1}{z} \frac{e^{z}-1}{e^{z}+1}\right] d z \\
= & \frac{1}{2} \int_{0}^{\infty} z^{k+1}\left(e^{z}+1\right)\left[e^{-(p+1) z}+e^{-(q+1) z}\right] \\
& \times\left[\frac{1}{(q-p) z} \tanh \frac{(q-p) z}{2}-\frac{1}{z} \tanh \frac{z}{2}\right] d z .
\end{aligned}
$$

Since the function $[\tanh (y / 2)] / y$ is even in $(-\infty, \infty)$ and decreasing in $(0, \infty)$, then

$$
\frac{1}{(q-p) z} \tanh \frac{(q-p) z}{2} \gtrless \frac{1}{z} \tanh \frac{z}{2}
$$

is valid for $|q-p|=|t-s| \lessgtr 1$. This reveals that $(-1)^{k}\left[\delta_{s, t}^{(k)}(x)-\delta_{s, t}^{(k)}(x+1)\right] \gtrless 0$ for $|t-s| \lessgtr 1$, which implies $(-1)^{k} \delta_{s, i}^{(k)}(x) \gtrless 0$ for $|t-s| \lessgtr 1$.

In conclusion, the function $\delta_{s, t}(x)$ is completely monotonic if $|t-s|<1$ and $-\delta_{s, t}(x)$ is completely monotonic if $|t-s|>1$. The proof of Theorem 1.1 is complete.

ProOF OF THEOREM 1.2. For real numbers $p, q \notin\{0,-1\}$, denote the function in the pair of braces in the ninth and tenth lines of $(2.5)$ by $T_{k}(p, q)$. Then

$$
\begin{aligned}
T_{k}(p, q)= & \left(\frac{1}{q-p}-1\right)\left\{\left[\frac{1}{p^{k+1}}-\frac{1}{q^{k+1}}\right]+\left[\frac{1}{(p+1)^{k+1}}-\frac{1}{(q+1)^{k+1}}\right]\right\} \\
& +2\left[\frac{1}{(p+1)^{k+1}}-\frac{1}{q^{k+1}}\right] \\
= & {[1-(q-p)]\left[\frac{1}{(p+1)^{k+1}(q+1)^{k+1}} \sum_{i=0}^{k}(q+1)^{i}(p+1)^{k-i}\right.} \\
& \left.+\frac{1}{p^{k+1} q^{k+1}} \sum_{i=0}^{k} q^{i} p^{k-i}\right]+\frac{2(q-p-1)}{(p+1)^{k+1} q^{k+1}} \sum_{i=0}^{k} q^{i}(p+1)^{k-i}
\end{aligned}
$$




$$
\begin{aligned}
= & (p-q+1)\left\{\frac{1}{(p+1)^{k+1}(q+1)^{k+1}} \sum_{i=0}^{k}(p+1)^{k-i}(q+1)^{i}\right. \\
& \left.+\frac{1}{p^{k+1} q^{k+1}} \sum_{i=0}^{k} p^{k-i} q^{i}-\frac{2}{(p+1)^{k+1} q^{k+1}} \sum_{i=0}^{k}(p+1)^{k-i} q^{i}\right\} \\
= & {[1-(q-p)]\left\{\sum_{i=0}^{k} \frac{1}{(p+1)^{i+1}(q+1)^{k-i+1}}\right.} \\
& \left.+\sum_{i=0}^{k} \frac{1}{p^{i+1} q^{k-i+1}}-2 \sum_{i=0}^{k} \frac{1}{(p+1)^{i+1} q^{k-i+1}}\right\} .
\end{aligned}
$$

(1) For $p>0$ and $q>0$, formula (2.5) tells us that $T_{k}(p, q) \gtrless 0$ for $|q-p| \lessgtr 1$. Considering (2.6), it is concluded that if $p>0$ and $q>0$ then the inequality

$$
\sum_{i=0}^{k} \frac{1}{(p+1)^{i+1}(q+1)^{k-i+1}}+\sum_{i=0}^{k} \frac{1}{p^{i+1} q^{k-i+1}}>2 \sum_{i=0}^{k} \frac{1}{(p+1)^{i+1} q^{k-\imath+1}}
$$

holds for $q-p>-1$ and reverses for $q-p<-1$.

(2) For $p<-1$ and $q<-1$, utilizing (2.3) reveals

$$
\begin{aligned}
T_{k}(p, q)= & (-1)^{k+1}\left\{\left(1+\frac{1}{q-p}\right) \frac{1}{[-(p+1)]^{k+1}}+\left(\frac{1}{q-p}-1\right) \frac{1}{(-p)^{k+1}}\right. \\
& \left.+\left(1-\frac{1}{q-p}\right) \frac{1}{[-(q+1)]^{k+1}}-\left(1+\frac{1}{q-p}\right) \frac{1}{(-q)^{k+1}}\right\} \\
= & \frac{(-1)^{k+1}}{k !} \int_{0}^{\infty} z^{k}\left\{e^{(p+1) z}-e^{p z}+\frac{e^{p z}-e^{q z}}{q-p}\right. \\
& \left.+e^{(q+1) z}-e^{q z}+\frac{e^{(p+1) z}-e^{(q+1) z}}{q-p}\right\} d z \\
= & \frac{(-1)^{k+1}}{k !} \int_{0}^{\infty} z^{k+1} e^{p z}\left(1+e^{z}\right)\left[1+e^{(q-p) z}\right] \\
& \times\left[\frac{1}{z} \frac{e^{z}-1}{e^{z}+1}-\frac{1}{(q-p) z} \frac{e^{(q-p) z}-1}{e^{(q-p) z}+1}\right] d z \\
= & \frac{(-1)^{k}}{k !} \int_{0}^{\infty} z^{k+1} e^{p z}\left(1+e^{z}\right)\left[1+e^{(q-p) z}\right] \\
& \times\left[\frac{1}{(q-p) z} \tanh \frac{(q-p) z}{2}-\frac{1}{z} \tanh \frac{z}{2}\right] d z .
\end{aligned}
$$

Thus $(-1)^{k} T_{k}(p, q) \gtrless 0$ for $|q-p| \lessgtr 1$. On combining this with (2.6), we deduce 
that if $p<-1$ and $q<-1$, then the inequalities

$$
\sum_{i=0}^{2 k} \frac{1}{(p+1)^{i+1}(q+1)^{2 k-i+1}}+\sum_{i=0}^{-2 k} \frac{1}{p^{i+1} q^{2 k-1+1}}>2 \sum_{i=0}^{2 k} \frac{1}{(p+1)^{i+1} q^{2 k-i+1}}
$$

and

$$
\sum_{i=0}^{2 k+1} \frac{1}{(p+1)^{i+1}(q+1)^{2 k-i+2}}+\sum_{i=0}^{2 k+1} \frac{1}{p^{i+1} q^{2 k-t+2}}<2 \sum_{i=0}^{2 k+1} \frac{1}{(p+1)^{i+1} q^{2 k-i+2}}
$$

hold for $q-p>-1$ and are reversed for $q-p<-1$.

(3) For $-1<p<0$ and $-1<q<0$, by using (2.3), we have

$$
\begin{aligned}
T_{k}(p, q)= & \left(1+\frac{1}{q-p}\right) \frac{1}{(p+1)^{k+1}}+\left(\frac{1}{q-p}-1\right) \frac{(-1)^{k+1}}{(-p)^{k+1}} \\
& +\left(1-\frac{1}{q-p}\right) \frac{1}{(q+1)^{k+1}}-\left(1+\frac{1}{q-p}\right) \frac{(-1)^{k+1}}{(-q)^{k+1}} \\
= & \frac{1}{k !} \int_{0}^{\infty} z^{k}\left(e^{q z}+e^{p z}\right)\left[\frac{1}{q-p} \frac{e^{(q-p) z}-1}{e^{(q-p) z}+1}+1\right]\left[e^{-(p+q+1) z}+(-1)^{k}\right] d z .
\end{aligned}
$$

If $k$ is even, then $T_{k}(p, q)>0$ and inequality (2.8) holds for all $-1<p<0$ and $-1<q<0$. If $k$ is odd, then $T_{k}(p, q) \gtrless 0$ for $p+q+1 \lessgtr 0$, and inequality (2.9) is valid for $p+q+1>0$ and is reversed for $p+q+1<0$.

(4) For $p<-1$ and $q>0$, using (2.3) shows

$$
\begin{aligned}
T_{k}(p, q)= & \left(1+\frac{1}{q-p}\right) \frac{(-1)^{k+1}}{[-(p+1)]^{k+1}}+\left(\frac{1}{q-p}-1\right) \frac{(-1)^{k+1}}{(-p)^{k+1}} \\
& +\left(1-\frac{1}{q-p}\right) \frac{1}{(q+1)^{k+1}}-\left(1+\frac{1}{q-p}\right) \frac{1}{q^{k+1}} \\
= & \frac{1}{k !} \int_{0}^{\infty} z^{k}\left(1+e^{z}\right) e^{p z}\left(\frac{e^{z}-1}{e^{z}+1}+\frac{1}{q-p}\right)\left[(-1)^{k+1}-e^{-(p+q+1) z}\right] d z .
\end{aligned}
$$

If $k$ is even, then $T_{k}(p, q)<0$ and inequality (2.8) holds for all $p<-1$ and $q>0$ by (2.6). If $k$ is odd, then $T_{k}(p, q) \gtrless 0$ for $p+q+1 \gtrless 0$, and inequality (2.9) holds for $p+q+1>0$ and reverses for $p+q+1<0$.

(5) For $p>0$ and $q<-1$, by using (2.3), we obtain

$$
\begin{aligned}
T_{k}(p, q)= & \left(1+\frac{1}{q-p}\right) \frac{1}{(p+1)^{k+1}}+\left(\frac{1}{q-p}-1\right) \frac{1}{p^{k+1}} \\
& +\left(1-\frac{1}{q-p}\right) \frac{(-1)^{k+1}}{[-(q+1)]^{k+1}}-\left(1+\frac{1}{q-p}\right) \frac{(-1)^{k+1}}{(-q)^{k+1}} \\
= & \frac{1}{k !} \int_{0}^{\infty} z^{k} e^{q z}\left(1+e^{z}\right)\left[\frac{e^{z}-1}{e^{z}+1}-\frac{1}{q-p}\right]\left[(-1)^{k+1}-e^{-(p+q+1) z}\right] d z .
\end{aligned}
$$


If $k$ is even, then $T_{k}(p, q)<0$ and inequality (2.8) is reversed for all $p>0$ and $q<-1$. If $k$ is odd, $T_{k}(p, q) \lessgtr 0$ for $p+q+1 \lessgtr 0$, and then the inequality (2.9) holds for $p+q+1<0$ and reverses for $p+q+1>0$.

(6) Letting $\theta>0$ and substituting $p=a / \theta$ and $q=b / \theta$ into (2.7) leads to

$$
\begin{aligned}
& \sum_{i=0}^{k} \frac{1}{(a / \theta+1)^{i+1}(b / \theta+1)^{k-i+1}}+\sum_{i=0}^{k} \frac{1}{(a / \theta)^{i+1}(b / \theta)^{k-i+1}} \\
& >2 \sum_{i=0}^{k} \frac{1}{(a / \theta+1)^{i+1}(b / \theta)^{k-i+1}}
\end{aligned}
$$

which is equivalent to (1.3).

Similarly, inequalities (1.4) and (1.5) can be deduced by substituting $p=a / \theta$ and $q=b / \theta$ into (2.8) and (2.9).

(7) Substituting $q=p-1$ into the first two lines in (2.6) leads immediately to $T_{k}(p, p-1)=0$, then inequalities (2.7)-(2.9) become equalities. Hence, if $b=a-\theta$ then inequalities (1.3)-(1.5) also become equalities. The proof of Theorem 1.2 is complete.

PROOF OF THEOREM 1.3. For $a>0$ and $b>0$, suppose inequality (1.3) holds for $b-a>-\theta$ and reverses for $b-a<-\theta$. If we assume $a=p \theta$ and $b=q \theta$ in (1.3), then simplifying yields that inequality (2.7) holds for $q-p>-1$ and reverses for $q-p<-1$, which is equivalent to $T_{k}(p, q) \gtrless 0$ for $|q-p| \lessgtr 1$. This means that $(-1)^{k}\left[\delta_{s, t}^{(k)}(x)-\delta_{s, t}^{(k)}(x+1)\right] \gtrless 0$ for $|t-s| \lessgtr 1$, which implies $(-1)^{k} \delta_{s, t}^{(k)}(x) \gtrless 0$ for $|t-s| \lessgtr 1$. Theorem 1.1 follows.

Conversely, if Theorem 1.1 is valid, then $(-1)^{k} \delta_{s, t}^{(k)}(x) \gtrless 0$ for $|t-s| \lessgtr 1$, which implies the function $(-1)^{k} \delta_{s, t}^{(k-1)}(x)$ is increasing/decreasing for $|t-s| \lessgtr 1$, and

$$
(-1)^{k} \delta_{s, t}^{(k-1)}(x)-(-1)^{k} \delta_{s, t}^{(k-1)}(x+1)=(-1)^{k}\left[\delta_{s, t}^{(k-1)}(x)-\delta_{s, t}^{(k-1)}(x+1)\right] \lessgtr 0
$$

for $|t-s| \lessgtr 1$, which means that $(-1)^{k}\left[\delta_{s, t}^{(k)}(x)-\delta_{s, t}^{(k)}(x+1)\right] \gtrless 0$ for $|t-s| \lessgtr 1$. Further, combining this with (2.5) and (2.6) leads to (2.7), and then (2.10). Inequality (1.3) is proved. The proof of Theorem 1.3 is complete.

\section{References}

[1] H. Alzer, "Sharp inequalities for the digamma and polygamma functions", Forum Math. 16 (2004) 181-221.

[2] N. Batir, "Some new inequalities for gamma and polygamma functions", J. Inequal. Pure Appl. Math. 6 (2005) no. 4, Art. 103; Available online at http://jipam.vu.edu.au/article.php?sid=577. RGMIA Res. Rep. Coll. 7 (2004), no. 3, Art. 1; Available online at http://rgmia.vu.edu.au/v7n3.html. 
[3] C. Berg, "Integral representation of some functions related to the gamma function", Mediterr. $J$. Math. 1 (2004) 433-439.

[4] Ch.-P. Chen, "Monotonicity and convexity for the gamma function", J. Inequal. Pure Appl. Math. 6 (2005) no. 4, Art. 100; Available online at http://jipam.vu.edu.au/article.php?sid=574.

[5] Á. Elbert and A. Laforgia, "On some properties of the gamma function", Proc. Amer. Math. Soc. 128 (2000) 2667-2673.

[6] N. Elezović, C. Giordano and J. Pečarić, "The best bounds in Gautschi's inequality", Math. Inequal. Appl. 3 (2000) 239-252.

[7] W. Gautschi, "Some elementary inequalities relating to the gamma and incomplete gamma function", J. Math. Phys. 38 (1959) 77-81.

[8] A. Z. Grinshpan and M. E. H. Ismail, "Completely monotonic functions involving the gamma and q-gamma functions", Proc. Amer. Math. Soc. 134 (2006) 1153-1160.

[9] D. Kershaw, "Some extensions of W. Gautschi's inequalities for the gamma function", Math. Comp. 41 (1983) 607-611.

[10] F. Qi, "The best bounds in Kershaw's inequality and two completely monotonic functions", $R G M I A$ Res. Rep. Coll. 9 (2006) no. 4, Art. 2; Available online at http://rgmia.vu.edu.au/v9n4.html.

[11] F. Qi, “Certain logarithmically $N$-alternating monotonic functions involving gamma and $q$-gamma functions", Nonlinear Funct. Anal. Appl. (2007) accepted. RGMIA Res. Rep. Coll., 8 (2005), 413-422; Available online at http://rgmia.vu.edu.au/v8n3.html.

[12] F. Qi, R.-Q. Cui, Ch.-P. Chen and B.-N. Guo, "Some completely monotonic functions involving polygamma functions and an application", J. Math. Anal. Appl. 310 (2005) 303-308.

[13] F. Qi and B.-N. Guo, "Complete monotonicities of functions involving the gamma and digamma functions", RGMIA Res. Rep. Coll. 7 (2004) 63-72; Available online at http://rgmia.vu.edu.au/v7n1.html.

[14] F. Qi, B.-N. Guo and Ch.-P. Chen, "The best bounds in Gautschi-Kershaw inequalities", Math. Inequal. Appl. 9 (2006) 427-436. RGMIA Res. Rep. Coll. 8 (2005), no. 2, Art. 17; Available online at http://rgmia.vu.edu.au/v8n2.html.

[15] F. Qi, B.-N. Guo and Ch.-P. Chen, "Some completely monotonic functions involving the gamma and polygamma functions", J. Austral. Math. Soc. 80 (2006) 81-88. RGMIA Res. Rep. Coll. 7 (2004), 31-36; Available online at http://rgmia.vu.edu.au/v7n1.html.

[16] H. van Haeringen, "Completely monotonic and related functions", Report 93-108, Faculty of Technical Mathematics and Informatics, Delft University of Technology, Delft, The Netherlands, 1993.

[17] D. V. Widder, The Laplace transform (Princeton University Press, Princeton, 1941). 